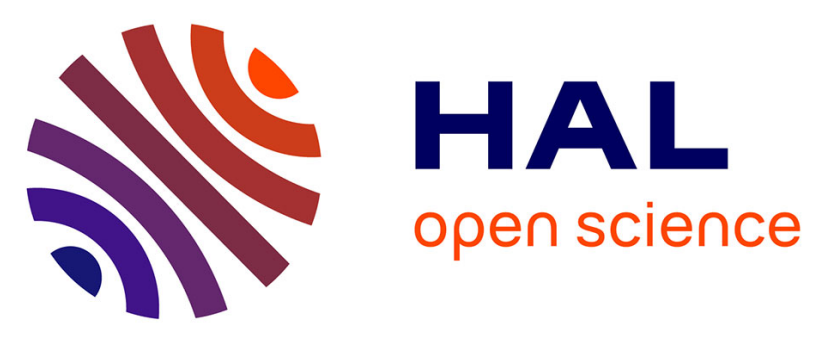

\title{
A Multi-agent Approach for Range Image Segmentation with Bayesian Edge Regularization
}

Smaine Mazouzi, Zahia Guessoum, Fabien Michel, Mohamed Batouche

\section{To cite this version:}

Smaine Mazouzi, Zahia Guessoum, Fabien Michel, Mohamed Batouche. A Multi-agent Approach for Range Image Segmentation with Bayesian Edge Regularization. ACIVS 2007 - 9th International Conference on Advanced Concepts for Intelligent Vision Systems, Aug 2007, Delft, Netherlands. pp.449460, 10.1007/978-3-540-74607-2_41. lirmm-00394193

\section{HAL Id: lirmm-00394193 \\ https://hal-lirmm.ccsd.cnrs.fr/lirmm-00394193}

Submitted on 23 Sep 2019

HAL is a multi-disciplinary open access archive for the deposit and dissemination of scientific research documents, whether they are published or not. The documents may come from teaching and research institutions in France or abroad, or from public or private research centers.
L'archive ouverte pluridisciplinaire HAL, est destinée au dépôt et à la diffusion de documents scientifiques de niveau recherche, publiés ou non, émanant des établissements d'enseignement et de recherche français ou étrangers, des laboratoires publics ou privés. 


\title{
A Multi-agent Approach for Range Image Segmentation with Bayesian Edge Regularization
}

\author{
Smaine Mazouzi ${ }^{1}$, Zahia Guessoum ${ }^{2}$, Fabien Michel $^{1}$, and Mohamed Batouche ${ }^{3}$ \\ ${ }^{1}$ MODECO-CReSTIC, Université de Reims, B.P. 1035, 51687, Reims, France \\ \{mazouzi,fmichel\}@leri.univ-reims.fr \\ ${ }^{2}$ LIP6, Université de Paris 6, 104, av. du Président Kennedy, 75016, Paris, France \\ zahia.guessoum@lip6.fr \\ ${ }^{3}$ Département d'informatique, Université de Constantine, 25000, Algérie \\ batouche@wissal.dz
}

\begin{abstract}
We present in this paper a multi-agent approach for range image segmentation. The approach consists in using autonomous agents for the segmentation of a range image in its different planar regions. Agents move on the image and perform local actions on the pixels, allowing robust region extraction and accurate edge detection. In order to improve the segmentation quality, a Bayesian edge regularization is applied to the resulting edges. A new Markov Random Field (MRF) model is introduced to model the edge smoothness, used as a prior in the edge regularization. The experimental results obtained with real images from the ABW database show a good potential of the proposed approach for range image analysis, regarding both segmentation efficiency, and detection accuracy.
\end{abstract}

Keywords: Image segmentation, Multi-agent systems, Range image, Bayesian-MRF estimation.

\section{Introduction}

Image segmentation consists in assigning pixels of an image to homogenous and disjoint sets called image regions. The segmentation of an image is often necessary to provide a compact and convenient description of its content, suitable for high level analysis and understanding. In range images, segmentation methods can be divided in two distinct categories: edge-based segmentation methods and region-based segmentation methods. In the first category, pixels which correspond to discontinuities in depth (jump edges) or in surface normals (roof edges) are selected and chained in order to delimit the regions in the image [611]. Edge-based methods are well known for their low computational cost; however, they are very sensitive to noise. Region-based methods use geometrical surface proprieties to gather pixels with the same properties in disjoint regions 51. Compared to edgebased methods, they are more stable and less sensitive to noise. However, they are computationally costly and their efficiency depends strongly on the selection of the region seeds. In both approaches, image denoising is often necessary. However, in the case of highly noisy images such as range images [8], a strong noise 
smoothing can erase roof edges and smooth edges. However, if the noise is undersmoothed distortions which remain in the image, lead to inaccurate or erroneous results. In range images, several recent segmentation methods fail because they do not correctly address and resolve this problem [101].

To deal with this problem, we introduce in this paper a multi-agent approach for range image segmentation. It consists in using a dense population of reactive agents. Agents move over the image and act on its pixels. While moving over the image, an agent adapts to the current planar region on which it is situated and memorizes its proprieties. At the boundaries between regions the agents will be in competition to align the pixels of the boundaries to their respective regions. The resulting alternative alignment of the boundary pixels preserves the region boundaries against erasing. A pixel is therefore processed according to both its neighborhood, and the agents that visit this pixel. An agent acts on the pixels with more certainty, acquired from its move on large areas on the regions of the image. The combination of the global information memorized within the agent, and the local information of the image provides more reliable decisions. Unfortunately, the competitive alignment of the region boundaries results in distorted and badly localized edges. So, these latter are corrected using a Bayesian regularization, based on a new Markov Random Field (MRF) model. The introduced MRF model is used to model the smoothness of image edges, considered as a prior in edge regularization. Extensive experimentations have been performed using real images from the ABW database 8]. The obtained results show a good potential of the proposed approach for an efficient and accurate segmentation of range images.

The remainder of the paper is organized as follows: In Section 2, we review some agent-based approaches for image segmentation, as well as some works having used Bayesian inference in range image segmentation. Section 3 is devoted to the proposed agent-based approach for range image segmentation. It describes the behavior of the agents and shows the underlying collective mechanism to deal with the image segmentation. In section 4, we introduce the Bayesian edge regularization. The experimental results are presented in Section 5, in which we discuss the parameter selection, and we analyze and comment the obtained results. Finally, a conclusion summarizes our contribution.

\section{Related Work}

\subsection{Agent-Based Systems for Image Segmentation}

Several agent-based systems have been proposed for image analysis and object recognition. In this review we consider only works which have addressed a solution in image segmentation.

Liu et al. 15] introduce a reactive agent-based system for brain MRI segmentation. Agents are used to label the pixels of the image according to their membership grade to the different regions. When finding pixels of a specific homogenous region, agents create offspring agents into their neighboring regions. An agent is created so that it becomes more likely to meet more homogenous 
pixels. For the same type of images, Richard et al. [16] propose a hierarchical architecture of situated and cooperative agents. Several control agents are distributed in the volume. The role of each one consists in creating tissue dedicated agents, which perform a local region growing. The statistical parameters of the data distribution, needed to perform region growing are updated according to the interaction between neighboring agents. Based on a cognitive architecture, Bovenkamp et al. 4 have developed a multi-agent system for IntraVascular UltraSound (IVUS) image segmentation. They aim to elaborate a high knowledgebased control over the algorithms of low-level image processing. In this system, an agent is assigned to every expected object in the image.

Most of the proposed agent-based systems for image segmentation are specific to image contents, and deal exclusively with jump edge detection. Following a supervised approach, these systems segment images in known and previously expected regions. The multi-agent approach proposed in this paper claims to be general and unsupervised. It aims to segment an image into its different regions by using geometrical surface proprieties. The adaptive and competitive behavior of the agents allows a collective and distributed image segmentation. We show in this work that simple interactions between agents can provide an alternative way for image segmentation.

\subsection{Bayesian Inference in Range Image Segmentation}

Few authors have integrated Bayesian inference in range image segmentation. Lavalle and Hutchinson 13 have used a Bayesian test to merge regions in both range and textured images. The merging of two regions depends on the probability that the resulting region is homogenous. Jain and Nadabar 9] have proposed a Bayesian method for edge detection in range images. Authors use the Line Process (LP) Markov random field (MRF) model 7] to label image pixels as EDGE or NON-EDGE pixels. Wang and Wang [17 have presented a hybrid scheme for range image segmentation. First, they proposed a joint Bayesian estimation of both pixel labels, and surface patches. Next, the solution is improved by combining the Scan Line algorithm 11, and the Multi-Level Logistic (MLL) MRF model [14. In spite of various contributions of the works previously cited, some aspects inherent to range image segmentation were omitted. Indeed, most of the works use Markovian models that are based exclusively on the surface smoothness prior. In our work, a refinement of the initial segmentation is performed by Bayesian regularization of the resulting region boundaries using a new Markov random field model. The latter models the edge smoothness, which is considered as a prior in the edge regularization.

\section{Multi-agent Range Image Segmentation}

\subsection{Surface Modeling}

A range image is a discretized two-dimensional array where at each pixel $(x, y)$ is recorded the distance $d(x, y)$ between the range finder and the corresponding 
point of the scene. Let $d^{*}(x, y)$ be the equation parameters of the tangent plane at $(x, y)$. The best tangent plane is obtained by the multiple regression method using neighbor pixels situated within a $3 \times 3$ window centred at $(x, y)$, and with close depths, according to a given threshold $\left(T r_{h}\right)$. The plane equation in a $3-D$ coordinate system may be expressed as follows: $z=a x+b y+c$; where $(a, b,-1)^{T}$ is a normal vector to the plane, and $|c| / \sqrt{a^{2}+b^{2}+1}$ is the orthogonal distance between the plane and the coordinate origin.

Two planes are considered equal if they have, according to some thresholds, the same orientation and the same distance to the coordinate origin. Let $\theta$ be the angle between the two normal vectors, and $h$ the distance between the two planes; so, the two planes are considered equal if $\sin (\theta) \leq \operatorname{Tr}_{\theta}$ and $h \leq T r_{h}$, where $\operatorname{Tr}_{\theta}$ and $T r_{h}$ are respectively the angle and the distance thresholds. Plane comparison is first used to test if a given pixel belongs to a planar region, given its plane equation. It is also used to test if the pixel is, or is not, a pixel of interest (edge or noise pixel). In this case, the pixel in question is considered as a pixel of interest if at least one of its neighbors has a different plane equation, according the previous thresholds.

\subsection{Agent Behavior}

The image is considered as the environment in which the agents are initialized at random positions. An agent checks if it is situated within a planar region, and adapts to this region if it is planar, by memorizing its plane equation. Next, the agent performs actions, which depend on both its state and the state of the pixel on which it is located. At each time $t$, an agent is characterized by its position $\left(x_{t}, y_{t}\right)$ over the image, and by its ability $A_{t}$ to act on the encountered pixels. At the beginning of the process, all the agents are unable to alter any pixel of the image. After having been adapted to a planar region, an agent becomes able to modify the first encountered pixel that not belongs to the current region $\left(A_{t}=\right.$ true). When an agent alters a pixel, it becomes unable to alter other pixels $\left(A_{t}=\right.$ false $)$ and starts again searching for a new planar region. An agent having modified a pixel records in an appropriate two-dimensional array $I$, at $\left(x_{t}, y_{t}\right)$ the last state of the visited pixel: $I\left(x_{t}, y_{t}\right) \in\{$ smoothed, aligned, unchanged $\}$. We show next, that this simple behavior of the agents allows both the detection of the edges, and the removal of the noise regions. Following are the tasks performed by an agent, according to its state and its position.

Searching for a Planar Region. After its creation, an agent randomly moves within the image and searches for a planar region around its current position. The agent uses a region seed formed by the last $P$ visited pixels. $P$ is called the adaptation path-length. It represents the confidence degree that the agent is situated within a planar region. So, the agent considers that it is within a planar region if the pixels of the seed form a planar surface. The agent memorizes the proprieties of the new region and considers it as its current planar region. Henceforth it becomes able to alter the first encountered pixel that does not belong to its new region ( $A_{t}=$ true). 
Moving on a Planar Region. While moving inside a planar region, an agent smoothes the image at the pixel on which it is located by updating the equations of both the memorized plane and the plane at the current position $\left(d^{*}\left(x_{t}, y_{t}\right)\right)$. This is done by replacing the two equations by their weighted average. Let $(a, b, c)$ and $\left(a^{\prime}, b^{\prime}, c^{\prime}\right)$ be the parameters respectively of the plane at the current pixel, and the memorized plane. Resulting parameters of the average plane are obtained as follows:

$$
\left(a^{\prime \prime}, b^{\prime \prime}, c^{\prime \prime}\right)=\frac{1}{1+p}\left(a+p a^{\prime}, b+p b^{\prime}, c+p c^{\prime}\right)
$$

where $p$ is the length of the path crossed by the agent on the current region.

Pixel Alignment. When an agent meets a pixel of interest (i.e. not belonging to its current planar region), the pixel is partially aligned to the planar region on which the agent moves. The parameters $\left(a^{\prime \prime}, b^{\prime \prime}, c^{\prime \prime}\right)$ of the new plane equation at the pixel position are obtained by linear combination of the current parameters $(a, b, c)$ and the parameters of the memorized plane equation $\left(a^{\prime}, b^{\prime}, c^{\prime}\right)$ :

$$
\left(a^{\prime \prime}, b^{\prime \prime}, c^{\prime \prime}\right)=\frac{1}{1+\xi}\left(a+\xi a^{\prime}, b+\xi b^{\prime}, c+\xi c^{\prime}\right)
$$

where $\xi$ is the alteration strength.

The agent becomes then unable to alter pixels $\left(A_{t}=\right.$ false $)$ and starts again to search for a new planar region. The alteration strength $\xi$ is a critical parameter which affects the quality of the results and the time of computation. Indeed, high values of $\xi$ lead to a fast detection of regions. However, the resulting region boundaries are strongly distorted and badly localized (Fig. 1b). Low values of $\xi$ result in a slow detection; nevertheless region boundaries in this case are well detected and localized (Fig. 1F). To speed up the segmentation process and avoid edge distortions, an agent chooses the alteration strength among $\xi_{\min }$ and $\xi_{\max }$ according to the information recorded by other agents in the array $I$. So, an agent assumes that the current planar region is adjacent to a noise region and thus uses $\xi_{\max }$ as alteration strength, if the number of "unchanged" pixels (situated in a noisy region) around the agent is greater than a certain threshold (fixed to 3 in our experimentations). Indeed, pixels labeled "unchanged" in the adjacent region mean that this latter is a noise region for which agents have not adapted and consequently have not smoothed its pixels. Otherwise, the agent assumes that the current planar region is adjacent to another one, where other agents have labeled the pixels as "smoothed" or "aligned". In this case the agent uses the alteration strength $\xi_{\text {min }}$.

\subsection{Edge Emergence and Noise Removal}

While moving over the image, an agent smoothes the pixels that approximately belong to its planar region, and it considers all other pixels as noise pixels. Among these latter, the agent systematically aligns the first encountered one to its current region. However, pixels on the boundaries of planar regions are true-edge pixels, and thus should not be aligned. Nevertheless, the competition 


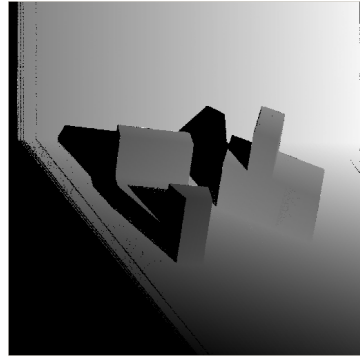

(a)

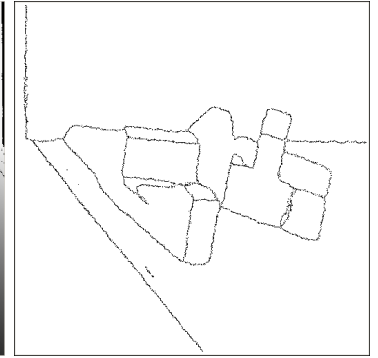

(b)

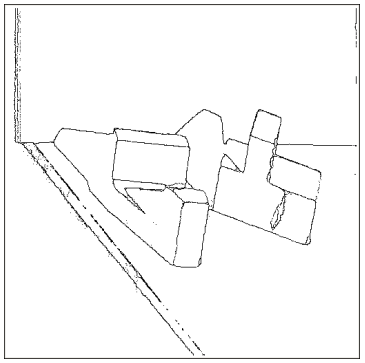

(c)

Fig. 1. The impact of the alteration strength on the segmentation results: (a) Range image (abw.test.3); (b) segmentation results with $\xi_{\min }=\xi_{\max }=4$ at $t=2500$; (c) segmentation results with $\xi_{\min }=0.3$ and $\xi_{\max }=5$ at $t=13000$

between agents preserves these pixels against an inappropriate smoothing. Indeed, around an edge between two adjacent planar regions, two groups of agents are formed on the two sides of the edge. Each group is formed of agents passing from one region to the other. Agents of each group align the pixels of the edge to their respective region. So, the pixels of the edge are continuously swapped between the two adjacent regions. The resulting alternative alignment of edge pixels allows these pixels to remain emergent in the image. This pattern of competitive actions between agents allows the emergence of the edges in the image, whose detection is not coded in any agent, but results from the collective action of all the agents.

An agent, having aligned a pixel which belongs to the border of a noise region and having moved inside this region, will not be able to adapt. Consequently, it cannot align any pixel when leaving the noise region. This occurs in two distinct situations: 1) when the region is planar but insufficiently large to allow agents to cross the minimal path-length $P$, necessary to be able to adapt; 2) when the region is sufficiently large but not planar, or made up of random depths (noise). In both situations, the agent leaves the noise region and will adapt inside the surrounding planar region. Boundaries of noise regions are continuously aligned from outside by including their pixels in the true surrounding regions. So, these regions continuously contract, and they finally disappear after several steps.

After several iterations (fixed to 13000), all image regions are well delimited by the detected boundaries. A simple region growing, steered by the detected boundaries, allows to provide the regions of the image.

\section{Bayesian Edge Regularization}

\subsection{Segmentation Modeling as Bayesian Estimation}

We have used piecewise smoothness of image edges as priors to model the distributions of boundary pixels in range images. Let $S$ denote the image lattice, and $M$ be the number of regions. So, each pixel in the image can take a label from the 
set of labels $L=\left\{l_{1}, . . l_{M}\right\}$. The labeling set $F=\left\{f_{(x, y)},(x, y) \in S, f_{(x, y)} \in L\right\}$, represents an image segmentation. If we assume that $F$ is Markovian, segmenting $S$ according to the Bayesian-MRF framework 14 can be done by computing the maximum a posteriori (MAP) $P(F / d)$ of the distribution of the set $F$, by considering $F$ as a Markov random field (MRF). According to Bayes rule, the maximum a posteriori $P(F / d)$ is expressed as follows:

$$
P(F / d)=\frac{p(d / F) P(F)}{p(d)}
$$

$P(F)=Z^{-1} e^{-U(F)}$ is the a priori probability of $F$, with $Z=\sum_{F} e^{-U(F)}$ a normalization constant called the partition function. The a priori energy $U(F)$ is the sum of clique potentials $V_{c}(F)$ over the set of all possible cliques $C$ : $U(F)=\sum_{c \in C} V_{c}(F)$.

In order to model the edge smoothness we use cliques formed by 9 sites located in a $3 \times 3$ window. Let $c_{3 \times 3}$ be a clique of $3 \times 3$ sites centred at an edge pixel $(x, y)$, and $\zeta(\zeta<0)$ a potential parameter. Considering all possible configurations in Fig. 2, the potential $V_{c}$ of cliques in $C$ can be expressed as follows:

$$
V_{c}\left(c_{3 \times 3}(x, y)\right)=\left\{\begin{array}{l}
\zeta \text { if } \exists\left(x^{\prime}, y^{\prime}\right),\left(x^{\prime \prime}, y^{\prime \prime}\right) \in c_{3 \times 3} \mid f_{(x, y)}=f_{\left(x^{\prime}, y^{\prime}\right)}=f_{\left(x^{\prime \prime}, y^{\prime \prime}\right)} \\
\text { and }\left(x^{\prime}, y^{\prime}\right), \overline{(x, y)},\left(x^{\prime \prime}, y^{\prime \prime}\right)=\pi \\
0 \text { if } \exists\left(x^{\prime}, y^{\prime}\right),\left(x^{\prime \prime}, y^{\prime \prime}\right) \in c_{3 \times 3} \mid f_{(x, y)}=f_{\left(x^{\prime}, y^{\prime}\right)}=f_{\left(x^{\prime \prime}, y^{\prime \prime}\right)} \\
\text { and }\left(x^{\prime}, y^{\prime}\right),(x, y),\left(x^{\prime \prime}, y^{\prime \prime}\right)=2 \pi / 3 \\
-\zeta \text { otherwise }
\end{array}\right.
$$

Configurations used to define $V_{c}$ depend on the surface type. For images containing polyhedral objects, considered in this work, $V_{c}$ is defined on the basis that the boundary between two adjacent regions is formed by pixels belonging to the same straight line (Fig. 2). So, configurations which correspond to locally unsmoothed edges are penalized by using a positive clique potential $(-\zeta)$.

The likelihood distribution $p(d / F)$, is obtained by assuming that observations $d$ are degraded by an independent Gaussian noise: $d(x, y)=a_{f_{(x, y)}} x+b_{f_{(x, y)}} y+$ $c_{f_{(x, y)}}+e(x, y) \cdot\left(a_{f_{(x, y)}}, b_{f_{(x, y)}}, c_{f_{(x, y)}}\right)$ are the parameters of the plane equation at the pixel $(x, y)$ assuming that it is labeled $f_{(x, y)}$.

$e(x, y) \sim N\left(0, \sigma_{l}^{2}\right)$ with $\sigma_{l}^{2}=\sum_{\left\{(x, y) \mid f_{(x, y)}=l\right\}}\left(a_{l} x+b_{l} y+c_{l}-d(x, y)\right)^{2}$. So the likelihood distribution is expressed as follows:

$$
p(d / F)=\frac{1}{\prod_{(x, y) \in S} \sqrt{2 \pi \sigma_{f_{(x, y)}^{2}}^{2}}} e^{-U(d / F)}
$$

with the likelihood energy $U(d / F)$ defined by:

$$
U(d / F)=\sum_{(x, y) \in S}\left(a_{f_{(x, y)}} x+b_{f_{(x, y)}} y+c_{f_{(x, y)}}-d(x, y)\right)^{2} / 2 \sigma_{f_{(x, y)}}^{2}
$$


Since $p(d)$ is constant for a fixed $d$, the solution $F^{*}$ is obtained by maximizing the a posteriori probability $P(F / d) \propto p(d / F) P(F)$, which is equivalent to minimizing the a posteriori energy $U(F / d)=U(d / F)+U(F)$ :

$$
F^{*}=\operatorname{argmin}\{U(d / F)+U(F)\}
$$

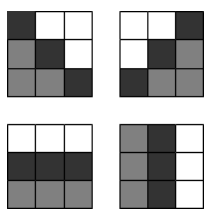

(a)
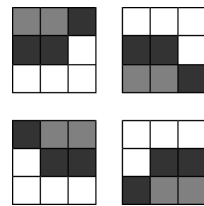

(b)
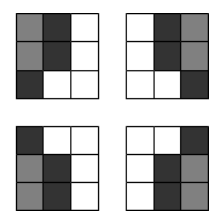

)

Fig. 2. Clique potential $V_{c}\left(c_{3 \times 3}\right)$ defined according to the edge smoothness prior. (a) Full smooth edge: $V_{c}\left(c_{3 \times 3}\right)=\zeta$; (b) partial smooth edge: $V_{c}\left(c_{3 \times 3}\right)=0$; otherwise, the edge is not locally smooth: $V_{c}\left(c_{3 \times 3}\right)=-\zeta$.

\subsection{Optimal Solution Computation}

By assuming that $F$ is Markovian, and the observations $\{d(x, y)\}$ are conditionally independent, we have used the Iterated Conditional Modes (ICM) algorithm [3] to minimize the a posteriori energy $U(F / d)$. By considering $U(F / d)$ as the sum of energies over all image sites: $U(F / d)=\sum_{(x, y) \in S} U\left(f_{(x, y)} / d(x, y)\right)$, we can separate it in two terms:

$$
U(F / d)=\sum_{(x, y) \in S^{\prime}} U\left(f_{(x, y)} / d(x, y)\right)+\sum_{(x, y) \in S-S^{\prime}} U\left(f_{(x, y)} / d(x, y)\right)
$$

where $S^{\prime}$ is the set of sites belonging to region boundaries:

$$
S^{\prime}=\left\{(x, y) \in S \mid \exists\left(x^{\prime}, y^{\prime}\right),\left(x^{\prime}-x, y^{\prime}-y\right) \in\{-1,0,1\}^{2} \wedge f_{(x, y)} \neq f_{\left(x^{\prime}, y^{\prime}\right)}\right\}
$$

Assuming the correctness of the labeling of the set $S-S^{\prime}$ (performed by the multiagent segmentation), the term $\sum_{(x, y) \in S-S^{\prime}} U\left(f_{(x, y)} / d(x, y)\right)$ is thus constant. So, minimizing the energy $U(F / d)$ is equivalent to minimizing the energy $U^{\prime}(F / d)$ which corresponds to the sites in $S^{\prime}: U^{\prime}(F / d)=\sum_{(x, y) \in S^{\prime}} U\left(f_{(x, y)} / d(x, y)\right)$.

The assumption of the correctness of the labeling of $S-S^{\prime}$ also allows us to define a constraint on the set of values that a site in $S^{\prime}$ can have during the execution of the ICM algorithm. Indeed, the label $f_{(x, y)}^{k}$ at the iteration $k$, of a site $(x, y)$ is chosen among the set $L^{\prime}(x, y) \subset L$ containing the labels of the sites in a $3 \times 3$ window centred at $(x, y)$. Formally, $L^{\prime}(x, y)$ is defined as follows:

$$
L^{\prime}(x, y)=\left\{l \mid \exists\left(x^{\prime}, y^{\prime}\right) \in S-S^{\prime},\left(x^{\prime}-x, y^{\prime}-y\right) \in\{-1,0,1\}^{2} \wedge f_{\left(x^{\prime}, y^{\prime}\right)}=l\right\}
$$

The two previous heuristics allow to speed up the calculation of the minimum of the a posteriori energy $U^{\prime}(F / d)$. They allow also to satisfy the region continuity constraint. For the latter problem, if we assume that the distance between two coplanar regions $R$ and $R^{\prime}$ is greater than 3 (size of the window), the labels $l_{R}$ and $l_{R^{\prime}}$ corresponding respectively to $R$ and $R^{\prime}$, cannot belong to the same set 
$L^{\prime}(x, y)$. For example, if the site $(x, y)$ is more close to $R$, it can not be labeled $l_{R^{\prime}}$, although energies $U^{\prime}\left(l_{R} / d(x, y)\right)$ and $U^{\prime}\left(l_{R^{\prime}} / d(x, y)\right)$ are equal.

\section{Experimentation and Analysis}

Hoover et al. have proposed a dedicated framework for the evaluation of range image segmentation algorithms [8], which has been used in several related works 1110151. The framework consists of a set of real range images, and a set of objective performance metrics. It allows to compare a machine-generated segmentation (MS) with a manually-generated segmentation, supposed ideal and representing the ground truth (GT). Region classification is performed according to a compare tool tolerance $T ; 50 \%<T \leq 100 \%$ which reflects the strictness of the classification. The 40 real images of the ABW set are divided into two subsets: 10 training images, and 30 test images. In our case, four methods, namely USF, WSU, UB and UE, cited in [8] are involved in the result comparison.

\subsection{Parameter Selection}

Since the evaluation framework provides a set of training images with ground truth segmentation (GT), we have opted to a supervised approach for the selection of parameters. For our approach, named 2ARIS for Agent-based Approach for Range Image Segmentation, seven parameters should be fixed: $\xi_{\min }, \xi_{\max }$, $\operatorname{Tr}_{\theta}, \operatorname{Tr}_{h}, N, P$, and $\zeta$. The performance criterion used in parameter selection is the average number of correctly detected regions with the compare tool tolerance $T$ set to $80 \%$. The set of parameters is divided into three subsets. 1) $\xi_{\text {min }}, \xi_{\max }, \operatorname{Tr}_{\theta}$, and $\operatorname{Tr}_{h}$ represent respectively the two alignment strengths, the thresholds of the angle, and the threshold of the depth. These parameters are used for testing and aligning pixels of the image. 2) $N$ and $P$ represent respectively the number of agents, and the adaptation path-length. These two parameters control the dynamic of the multi-agent system. 3) $\zeta$ represents the clique potential parameter. For the first parameter subset, 256 combinations namely $\left(\xi_{\text {min }}, \xi_{\text {max }}, \operatorname{Tr}_{\theta}, T r_{h}\right) \in\{0.5,0.3,0.1,0.05\} \times\{1.0,3.0,5.0,7.0\} \times$ $\left\{15^{\circ}, 18^{\circ}, 21^{\circ}, 24^{\circ}\right\} \times\{12,16,20,24\}$ were run on the training images. These parameters are set as follows: $\xi_{\min }=0.3, \xi_{\max }=5.0, \operatorname{Tr}_{\theta}=21^{\circ}$ and $T r_{h}=16$. In order to set the parameters $N$ and $P, 25$ combinations of these parameters, namely $(N, P) \in\{1500,2000,2500,3000,3500\} \times\{3,5,7,9,11\}$ were run on the training set. Optimal values of $N$ and $P$ are respectively 2500 and 7 . The Coding method [2] was used to estimate the parameter $\zeta$. A value of $\zeta$ is computed for each image in the training set. The Average is used as the final value of the parameter. The optimum for each training image is calculated by the simulated annealing algorithm [12, using a Gibbs sampler [7]. The average value of $\zeta$ obtained with the used training set is $-0.27 \times 10^{-4}$.

\subsection{Experimental Results}

Fig. 3 shows an instance of segmentation progression within time of a typical range image (abw.test.8) [85]. The time $t$ represents the number of steps 
performed by each agent since the beginning of the process. Figures $3 \mathrm{~b}, 3 \mathrm{k}, \mathrm{3} \mathrm{d}$ and 3. show the set of pixels of interest (edge or noise pixels) respectively at $t=1000,5000,9000$ and 13000 . Regions are progressively smoothed by aligning noise pixels to the surrounding planar regions. Edges between adjacent regions are also progressively thinned. At the end of the process, region borders consist of thin lines of one pixel wide (Fig. 3 $\mathrm{k}$ ). Fig. 3ir shows the segmentation result after edge regularization. We can note that the positions of some edge pixels have been corrected. The regularization was performed typically for roof edges, situated between adjacent regions.

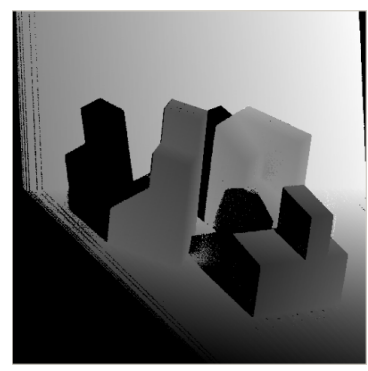

(a)

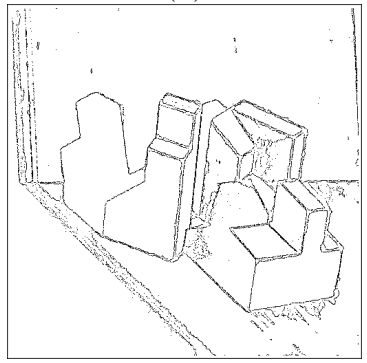

(d)

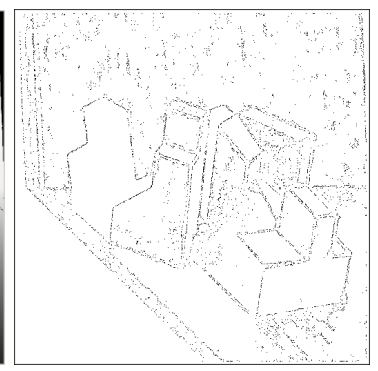

(b)

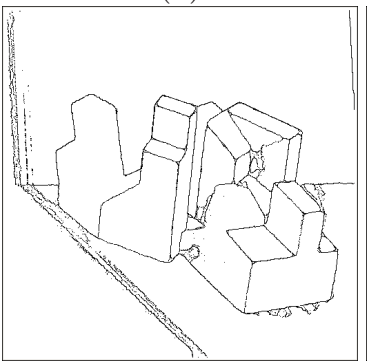

(e)

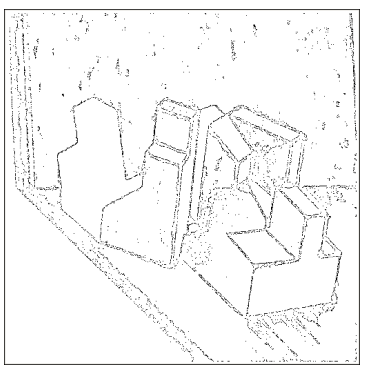

(c)

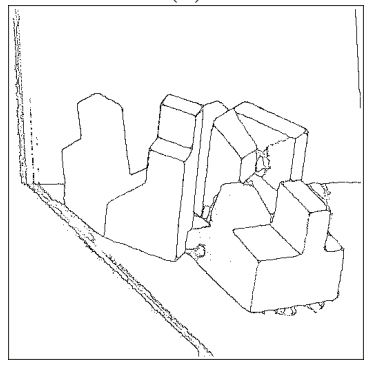

(f)

Fig. 3. Segmentation progression. (a) Range image (abw.test.8) ; (b) at $t=1000$; (c) at $t=5000 ;(\mathrm{d})$ at $t=9000$; (e) at $t=13000$; (f) after edge regularization

Table 1 contains the average results obtained with all test images, and for all performance metrics. The compare tool tolerance was set to the typical value $80 \%$. By considering both correct detection and incorrect detection metrics, obtained results show the good efficiency of our method. Fig. 4 shows the average numbers of correctly detected regions for all test images, according to the compare tool tolerance $T$. Results show that the number of correctly detected regions by our system is in average better than those of USF, UB and WSU. For instance, our system scored higher than WSU for all the values of the compare tool tolerance $T$. It scored higher than USF for $T \geq 80 \%$, and better than UB for $T \leq 80 \%$. For all incorrect detection metrics (instances of Over-segmentation, 
Table 1. Average results of the different involved methods with $T=80 \%$

\begin{tabular}{ccccccc}
\hline \multicolumn{3}{c}{ Method } & GT & Correct det. Over-seg. Under-seg. & Missed & Noise \\
\hline USF & 15.2 & 12.7 & 0.2 & 0.1 & 2.1 & 1.2 \\
WSU & 15.2 & 9.7 & 0.5 & 0.2 & 4.5 & 2.2 \\
UB & 15.2 & 12.8 & 0.5 & 0.1 & 1.7 & 2.1 \\
UE & 15.2 & 13.4 & 0.4 & 0.2 & 1.1 & 0.8 \\
2ARIS & 15.2 & 13.0 & 0.5 & 0.1 & 1.4 & 0.9 \\
\hline
\end{tabular}

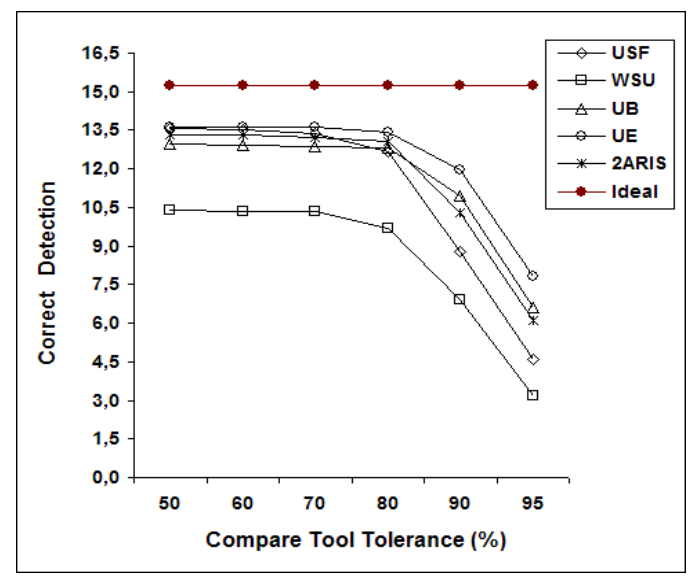

Fig. 4. Average results of correctly detected regions of all methods, according to the compare tool tolerance $T ; 0.5<T \leq 1.0$

Under-segmentation, Missed Region, Noise Region), our system has equivalent scores to those of UE and USF. The two latter scored higher than UB and WSU, regarding incorrect detection metrics.

\section{Conclusion}

In this paper we have presented a multi-agent approach for range image segmentation. Edge detection and noise removal have resulted from indirect interaction between autonomous agents moving over the image. Image edges, for which no explicit detection was coded in any agent, result from the collective action of all the agents. The proposed approach aims to improve efficiency and to deal with the problem of result accuracy. Indeed, obtained results are better than those provided by the traditional region growing algorithm. Bayesian edge regularization using an appropriate MRF model, introduced in this paper, has allowed improving the segmentation results. The experimental results obtained with real images from the ABW database were compared to those provided by four typical algorithms for range image segmentation. Comparison results show the good efficiency of the proposed approach for accurate segmentation of range images. 


\section{References}

1. Bab Hadiashar, A., Gheissari, N.: Range image segmentation using surface selection criterion. IEEE Transactions on Image Processing 15(7), 2006-2018 (2006)

2. Besag, J.E.: Spatial interaction and statistical analysis of lattice systems. Journal of the Royal Statistical Society, Series B 36, 192-236 (1974)

3. Besag, J.E.: On the statistical analysis of dirty pictures. Journal of the Royal Statistical Society, Series B 48, 259-302 (1986)

4. Bovenkamp, E.G.P., Dijkstra, J., Bosch, J.G., Reiber, J.H.C.: Multi-agent segmentation of IVUS images. Pattern Recognition 37(4), 647-663 (2004)

5. Ding, Y., Ping, X., Hu, M., Wang, D.: Range image segmentation based on randomized hough transform. Pattern Recognition Letters 26(13), 2033-2041 (2005)

6. Fan, T.J., Medioni, G.G., Nevatia, R.: Segmented description of 3-D surfaces. IEEE J. Robotics Automat. 3(6), 527-538 (1987)

7. Geman, S., Geman, D.: Stochastic relaxation, Gibbs distributions, and the Bayesian restoration of images. IEEE Transactions on Pattern Analysis and Machine Intelligence 6(6), 721-741 (1984)

8. Hoover, A., Jean-Baptiste, G., Jiang, X., Flynn, P.J., Bunke, H., Goldgof, D.B., Bowyer, K.W., Eggert, D.W., Fitzgibbon, A.W., Fisher, R.B.: An experimental comparison of range image segmentation algorithms. IEEE Transactions on Pattern Analysis and Machine Intelligence 18(7), 673-689 (1996)

9. Jain, A.K., Nadabar, S.G.: MRF model-based segmentation of range images. In: International Conference on Computer Vision, pp. 667-671 (1990)

10. Jiang, X., Bowyer, K.W., Morioka, Y., Hiura, S., Sato, K., Inokuchi, S., Bock, M., Guerra, C., Loke, R.E., Hans du Buf, J.M.: Some further results of experimental comparison of range image segmentation algorithms. In: International Conference on Pattern Recognition, vol. 4, pp. 4877-4882 (2000)

11. Jiang, X., Bunke, H.: Edge detection in range images based on Scan Line approximation. Computer Vision and Image Understanding 73(2), 183-199 (1999)

12. Kirkpatrick, J.S., Gelatt, Jr. C.D., Vecchi, M.P.: Optimization by simulated annealing. Readings in computer vision: issues, problems, principles, and paradigms, pp. 606-615 (1987)

13. LaValle, S.M., Hutchinson, S.A.: Bayesian region merging probability for parametric image models. In: Proc. 1993 IEEE Conference on Computer Vision and Pattern Recognition, pp. 778-779. IEEE Computer Society Press, Los Alamitos (1993)

14. Li, S.Z.: Markov random field modeling in image analysis. Springer, New York, Inc. Secaucus, NJ, USA (2001)

15. Liu, J., Tang, Y.Y.: Adaptive image segmentation with distributed behavior-based agents. IEEE Transactions on Pattern Analysis and Machine Intelligence 21(6), 544-551 (1999)

16. Richard, N., Dojat, M., Garbay, C.: Automated segmentation of human brain MR images using a multi-agent approach. Artificial Intelligence in Medicine 30(2), 153 $176(2004)$

17. Wang, X., Wang, H.: Markov random field modeled range image segmentation. Pattern Recognition Letters 25(3), 367-375 (2004) 